\title{
COMPUTING THE DISCRIMINANTS OF BRAUER'S CENTRALIZER ALGEBRAS
}

\author{
PHIL HANLON AND DAVID WALES
}

\begin{abstract}
This paper discusses a computational problem arising in the study of the structure theory of Brauer's orthogonal and symplectic centralizer algebras. The problem is to compute the ranks of certain combinatorially defined matrices $Z_{m, k}(x)$ (these matrices are presented in $\S 2$ ). This computation is difficult because the sizes of the matrices $Z_{m, k}(x)$ are enormous even for small values of $m$ and $k$. However, there is a great deal of symmetry amongst the entries of the matrices. In this paper we show how to design algorithms that take full advantage of this symmetry, using the representation theory of the symmetric groups. We also present data collected using these algorithms and a number of conjectures about the centralizer algebras.
\end{abstract}

\section{INTRODUCTION}

Early in this century invariant theorists began to study the commuting algebras of the tensor powers of the defining representations for the classical groups (see Weyl [19]). These algebras are defined in the following way. Let $G$ be a classical group, let $V$ be its defining representation, and let $T^{f} V$ be the $f$ th tensor power of $V$. The group action of $G$ on $V$ lifts to the diagonal action of $G$ on $T^{f} V$, defined by $g \cdot\left(v_{1} \otimes v_{2} \otimes \cdots \otimes v_{f}\right)=\left(g v_{1}\right) \otimes\left(g v_{2}\right) \otimes \cdots \otimes\left(g v_{f}\right)$. Define the commuting algebra, $\operatorname{End}_{G}\left(T^{f} V\right)$, of this action to be the algebra of all linear transformations of $T^{f} V$ which commute with this action of $G$.

The first important result was due to Schur [16] who studied the $G=$ $\mathrm{Gl}(n, \mathbb{C})$ case. He showed that there is a surjective algebra homomorphism from $\mathbb{C} \operatorname{Sym}(f)$ onto $\operatorname{End}_{\mathrm{Gl}(n, \mathbb{C})}\left(T^{f} \mathbb{C}^{n}\right)$, which is an isomorphism for $f \leq n$. He went on to identify the kernel of this homomorphism, thus giving a complete description of the centralizer algebra $\operatorname{End}_{\mathrm{Gl}(n, \mathbb{C})}\left(T^{f} \mathbb{C}^{n}\right)$.

The next cases considered were the orthogonal group, $G=\mathrm{O}(n, \mathbb{R})$, and the symplectic group $\operatorname{Sp}(2 n, \mathbb{R})$. In a 1937 paper, Richard Brauer [3] defined two

Received March 5, 1987; revised July 30, 1987, December 28, 1987, and May 8, 1989.

1980 Mathematics Subject Classification (1985 Revision). Primary 22E46.

The work of the first author was partially supported by NSF grant number MCS-8401718, and was mainly done while this author was a Bantrell Fellow at The California Institute of Technology.

The work of the second author was partially supported by the NSF. 
algebras $\mathscr{A}_{f}^{(x)}$ and $\mathscr{B}_{f}^{(x)}$ indexed by a positive integer $f$ and a real indeterminate $x$. He also constructed surjective algebra homomorphisms

$$
\varphi_{f}^{(n)}: \mathscr{A}_{f}^{(n)} \rightarrow \operatorname{End}_{\mathrm{O}(n, \mathbb{R})}\left(T^{f} \mathbb{R}^{n}\right)
$$

and

$$
\psi_{f}^{(2 m)}: \mathscr{B}_{f}^{(2 m)} \rightarrow \operatorname{End}_{\mathrm{Sp}(2 m, \mathbb{R})}\left(T^{f} \mathbb{R}^{2 m}\right)
$$

and he showed that these homomorphisms are isomorphisms if $n$ and $m$ are large enough. He failed to give a description of the kernels of the maps $\varphi_{f}^{(n)}$ and $\psi_{f}^{(2 m)}$ in the cases where these maps are not isomorphisms.

In the hope of finding an explicit description of these kernels, the present authors began the study of the algebra structures of $\mathscr{A}_{f}^{(x)}$ and $\mathscr{B}_{f}^{(x)}$ for $x$ an arbitrary real. One simplification of the problem comes in noting that the algebras $\mathscr{A}_{f}^{(x)}$ and $\mathscr{B}_{f}^{(-x)}$ are isomorphic. So it was only necessary to study the algebra $\mathscr{A}_{f}^{(x)}$. The authors hoped to be able to describe the radical of $\mathscr{A}_{f}^{(x)}$ and the matrix ring decomposition of $\mathscr{A}_{f}^{(x)} / \operatorname{Rad}\left(\mathscr{A}_{f}^{(x)}\right)$.

In an earlier paper [7], the authors found the matrix ring decomposition of $\mathscr{A}_{f}^{(x)} / \operatorname{Rad}\left(\mathscr{A}_{f}^{(x)}\right)$ and reduced the problem of finding the radical of $\mathscr{A}_{f}^{(x)}$ to the problem of computing the ranks of certain combinatorially defined matrices $Z_{m, k}(x)$ which are described in the next section. This paper concerns the computation of these ranks and the slightly weaker problem of computing the determinants of $Z_{m, k}(x)$. These determinants, when considered in an appropriate way, are discriminants of the Brauer algebras $\mathscr{A}_{f}^{(x)}$.

The computational methods described in this paper make strong use of the representation theory of the symmetric groups. The paper is organized as follows: $\S 2$ describes in combinatorial terms the computations that need to be done, $\S 3$ gives background information about representation theory, $\S 4$ explains how to use this representation theory to construct algorithms, $\S 5$ contains the result of computations that have been done by the authors, and $\$ 6$ contains previous results about the radicals of the $\mathscr{A}_{f}^{(x)}$ as well as a number of conjectures that are suggested by the data in $\S 5$.

We will assume familiarity with the standard notation, terminology, and theorems from the representation theory of the symmetric groups. In particular, the reader will need to know the definition of a Littlewood-Richardson filling and the Littlewood-Richardson rule (see James [9, pp. 51-64] or Macdonald [14, pp. 68-73]). We write $g_{\lambda \mu \eta}$ for the number of Littlewood-Richardson fillings of $[\lambda / \mu]$ having content $\eta$, and we write $\lambda \vdash l$ to signify that $\lambda$ is a partition of $l$. Other notation and terminology can be found in Macdonald [14]. 


\section{The COMPUTATIONAL PROBLEM}

In this section we will define a class of square matrices $Z_{m, k}(x)$ which are indexed by positive integers $m$ and $k$ and which have entries that are polynomials in $x$. Our computational problem will be to compute the rank of $Z_{m, k}(x)$ for every complex number $x$. The determinant of $Z_{m, k}(x)$ is known to be nonzero as a polynomial in $x$, so the rank of $Z_{m, k}(x)$ is full except at a finite number of values of $x$ (those $x$ that are roots of $\operatorname{det}\left(Z_{m, k}(x)\right)$ ). So our computational problem breaks into two parts:

(1) Compute the roots of $\operatorname{det}\left(Z_{m, k}(x)\right)$.

(2) For each root $r$, compute the rank of $Z_{m, k}(r)$.

Definition 2.1. Let $m$ and $k$ be nonnegative integers. An $m, k$ partial 1-factor is a graph with $m+2 k$ points and $k$ lines which satisfies:

$\langle\mathrm{C} 1\rangle$ Every point has degree 0 or 1 .

(C2) The $m$ points of degree 0 are labelled with the numbers $1,2, \ldots, m$.

We always use $f$ to denote $m+2 k$, and we use lower case Greek letters $\delta, \delta_{1}, \delta_{2}, \ldots$ to denote partial 1 -factors. The points of degree 0 in a partial 1-factor $\delta$ are called the free points of $\delta$. Lastly, we let $B_{m, k}$ denote the set of $m, k$ partial 1-factors and we let $V_{m, k}$ be the complex vector space with basis $B_{m, k}$. The notion of a partial 1-factor was introduced in [7], where there is a discussion of how they are related to the Brauer centralizer algebras.

Let $\delta_{1}$ and $\delta_{2}$ be elements of $B_{m, k}$. It is easy to check that the union of $\delta_{1}$ and $\delta_{2}$ is a graph consisting of some number $\gamma\left(\delta_{1}, \delta_{2}\right)$ of cycles together with $m$ paths $P_{1}, \ldots, P_{m}$. If $u$ is an endpoint of $P_{i}$, then $u$ is a free point of either $\delta_{1}$ or $\delta_{2}$. Hence, the endpoints of each path are labelled. We say $\delta_{1}$ and $\delta_{2}$ are consistent if each path of $\delta_{1} \cup \delta_{2}$ has the property that its endpoints have the same label. Otherwise, $\delta_{1}$ and $\delta_{2}$ are inconsistent.

Definition 2.2. Let $m$ and $k$ be nonnegative integers. Define a matrix $Z_{m, k}(x)$ with rows and columns indexed by $B_{m, k}$. For $\delta_{1}, \delta_{2} \in B_{m, k}$, let the $\delta_{1}, \delta_{2}$ entry of $Z_{m, k}(x)$ be

$$
\left(Z_{m, k}(x)\right)_{\delta_{1}, \delta_{2}}= \begin{cases}x^{\gamma\left(\delta_{1}, \delta_{2}\right)} & \text { if } \delta_{1} \text { and } \delta_{2} \text { are consistent } \\ 0 & \text { if } \delta_{1} \text { and } \delta_{2} \text { are inconsistent }\end{cases}
$$

Note that each diagonal entry of $Z_{m, k}(x)$ is $x^{k}$ and that every off-diagonal entry is either 0 or $x^{e}$ with $e<k$. So the determinant of $Z_{m, k}(x)$ is a nonzero polynomial in $x$ of degree $k\left|B_{m, k}\right|$.

As an example, consider $f=4$ and $m=2$. In this case, the matrix $Z_{m, k}(x)$ 
is 12 by 12 . An ordered basis for $B_{m, k}$ is the set below:

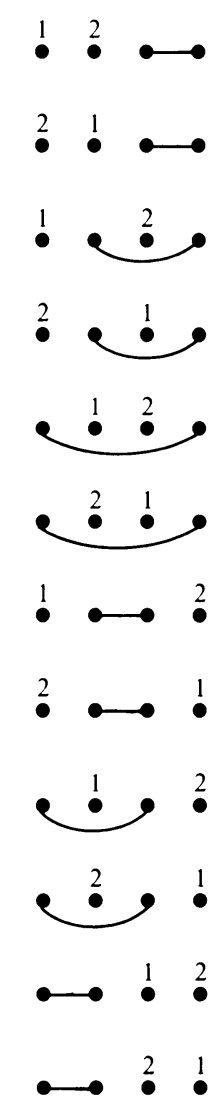

The matrix $Z_{m, k}(x)$ with respect to this basis is given by

$$
\left[\begin{array}{llllllllllll}
x & 0 & 1 & 0 & 0 & 1 & 1 & 0 & 0 & 1 & 0 & 0 \\
0 & x & 0 & 1 & 1 & 0 & 0 & 1 & 1 & 0 & 0 & 0 \\
1 & 0 & x & 0 & 1 & 0 & 1 & 0 & 0 & 0 & 0 & 1 \\
0 & 1 & 0 & x & 0 & 1 & 0 & 1 & 0 & 0 & 1 & 0 \\
0 & 1 & 1 & 0 & x & 0 & 0 & 0 & 1 & 0 & 0 & 1 \\
1 & 0 & 0 & 1 & 0 & x & 0 & 0 & 0 & 1 & 1 & 0 \\
1 & 0 & 1 & 0 & 0 & 0 & x & 0 & 1 & 0 & 1 & 0 \\
0 & 1 & 0 & 1 & 0 & 0 & 0 & x & 0 & 1 & 0 & 1 \\
0 & 1 & 0 & 0 & 1 & 0 & 1 & 0 & x & 0 & 1 & 0 \\
1 & 0 & 0 & 0 & 0 & 1 & 0 & 1 & 0 & x & 0 & 1 \\
0 & 0 & 0 & 1 & 0 & 1 & 1 & 0 & 1 & 0 & x & 0 \\
0 & 0 & 1 & 0 & 1 & 0 & 0 & 1 & 0 & 1 & 0 & x
\end{array}\right]=Z_{2,1}(x)
$$

As stated earlier, our computational problem is to first compute the roots of $\operatorname{det}\left(Z_{m, k}(x)\right)$ and then for each root $r$ to compute the rank of $Z_{m, k}(r)$. This appears to be an intractable computation for all but a few small values of $m$ and $k$. It is easy to check that the size of $B_{m, k}$ (which is the length of each 
row and column of $\left.Z_{m, k}(x)\right)$ is

$$
\left|B_{m, k}\right|=(m+2 k) ! / 2^{k} k ! .
$$

Even for small values of $m$ and $k$, the matrices $Z_{m, k}(x)$ are very large. Moreover, they have entries which are polynomials in $x$, and they are not particularly sparse.

However, there is a great deal of symmetry amongst the entries of $Z_{m, k}(x)$. We will exploit this symmetry to design algorithms which will carry out our computation for some surprisingly large values of $m$ and $k$. Our first step is to express the symmetries more explicitly.

There are permutation actions of both $\operatorname{Sym}(f)$ and $\operatorname{Sym}(m)$ on the set $B_{m, k}$. A permutation $\sigma$ in $\operatorname{Sym}(f)$ acts on a 1 -factor $\delta$ by permuting the $f$ points of $\delta$ and correspondingly permuting the vertex labels and edges. To be precise, if $u$ is joined to $v$ in $\delta$, then $\sigma u$ is joined to $\sigma v$ in $\sigma \delta$, and if $z$ is a free point of $\delta$ with label $a$, then $\sigma z$ is a free point of $\sigma \delta$ with label $a$. The group $\operatorname{Sym}(m)$ acts on $B_{m, k}$ by changing the values of the labels. If $\pi$ is in $\operatorname{Sym}(m)$ and $u$ is a free point of $\delta$ with label $a$, then $u$ is a free point of $\pi \delta$ with label $\pi a$. The edges of $\delta$ and $\pi \delta$ are identical.

It is easy to see that these actions of $\operatorname{Sym}(m)$ and $\operatorname{Sym}(f)$ commute. Also, it is straightforward to check that $Z_{m, k}(x)$ commutes with the actions of $\operatorname{Sym}(f)$ and $\operatorname{Sym}(m)$. Hence we have the following theorem.

Theorem 2.3. The matrix $Z_{m, k}(x)$ commutes with the action of $\operatorname{Sym}(f) \times$ $\operatorname{Sym}(m)$ on $V_{m, k}$.

Theorem 2.3 tells us that many of the entries of $Z_{m, k}(x)$ are identical, a fact which we would like to exploit when we compute the determinant of $Z_{m, k}(x)$. In the next section we review some facts about the representation theory of finite groups, and we state a theorem which tells us how to take full advantage of the symmetries expressed by Theorem 2.3 .

\section{A TOOL FROM REPRESENTATION THEORY}

In this section we discuss a theorem from representation theory which will be the basis of our algorithm. To understand the statement and application of this result, the reader will need some background in the representation theory of finite groups. There are many excellent sources for this information, in particular the books by Feit [6], Boerner [2], Curtis and Reiner [4], and Ledermann [12]. The reader will need a more sophisticated background in the representation theory of the symmetric groups. We recommend the books by James [9] and James and Kerber [10].

We now state the main result of this section, which can be thought of as a constructive form of Schur's Lemma. This is a well-known result in representation theory, although it is usually not stated in this kind of algorithmic form. The proof of this theorem is straightforward and we leave it to the reader. 
Theorem 3.1. Let $G$ be a finite group with irreducible representations $\phi_{1}, \ldots$, $\phi_{c}$. Let $\phi$ be a representation of $G$ on a complex vector space $V$ which decomposes into irreducibles as

$$
\phi=\sum_{i=1}^{c} m_{i} \phi_{i} .
$$

Let $Z$ be a linear transformation of $V$ which commutes with the action of $G$. Then $Z$ is similar to a matrix which is a direct sum over $i$ of matrices $Z_{i}$, where $Z_{i}$ is an $m_{i} \times m_{i}$ matrix repeated in the direct sum $\operatorname{deg}\left(\phi_{i}\right)$ times. Moreover, $Z_{i}$ can be computed as follows:

Step 1: Choose a complete set of primitive orthogonal idempotents $\left\{e_{v}^{(u)}: 1 \leq u \leq c, 1 \leq v \leq \operatorname{deg}\left(\phi_{u}\right)\right\}$ in the group algebra $\mathbb{C} G$.

Step 2: Find $m_{i}$ vectors $v_{1}, \ldots, v_{m_{i}} \in V$ such that $\phi\left(e_{1}^{(i)}\right) v_{1}, \ldots$, $\phi\left(e_{1}^{(i)}\right) v_{m_{i}}$ are linearly independent.

Step 3: Let $V_{1}^{(i)}$ be the subspace of $V$ spanned by $\phi\left(e_{1}^{(i)}\right) v_{1}, \ldots$, $\phi\left(e_{1}^{(i)}\right) v_{m_{i}}$. The space $V_{1}^{(i)}$ is $Z$-invariant and $Z_{i}$ is the restriction of $Z$ to $V_{1}^{(i)}$.

Remark. Any multiple of $e_{1}^{(i)}$ will do just as well.

There are two difficulties one encounters implementing the algorithm set out in Theorem 3.1. The first problem is to obtain a complete set of primitive orthogonal idempotents for the particular group $G$ under consideration. This can be an insurmountable problem since these idempotents are in practice very difficult to compute. However, idempotents are known for some groups, and in particular for many groups that are likely to come up in practice. For our application we will need idempotents for the symmetric groups. These have been known since the time of Alfred Young. We present these idempotents and discuss some of their combinatorial properties below.

The second problem one encounters with this algorithm is how to find the vectors $v_{1}, \ldots, v_{m_{i}} \in V$ such that $\phi\left(e_{1}^{(i)}\right) v_{1}, \ldots, \phi\left(e_{1}^{(i)}\right) v_{m_{i}}$ are linearly independent. This problem depends on the particular representation $\phi$ under consideration. The authors know of no general tools for finding these vectors.

We described Theorem 3.1 as a constructive form of Schur's Lemma. That comes from considering the case where $\phi=\phi_{i}$ is irreducible. In this case, Schur's Lemma tells us that any matrix $Z$ which commutes with $\phi$ is a scalar matrix. One can compute the scalar by comparing $v$ to $Z v$ for any nonzero vector $v$. Theorem 3.1 generalizes this idea to representations $\phi$ which are not irreducible.

In our applications of Theorem 3.1 the group $G$ will be either a symmetric group or a direct product of symmetric groups. We end this section with a brief description of the primitive orthogonal idempotents we will use for these groups. 
Let $G$ be the symmetric group $\operatorname{Sym}(f)$. It is well known that there is an indexing of the irreducible representations of $G$ by partitions $\lambda$ of $f$ such that the dimension of the irreducible $\chi^{\lambda}$ corresponding to $\lambda$ is the number of standard Young tableaux (SYT) of shape $\lambda$. If $t$ is a standard Young tableau of shape $\lambda$, let $C_{t}$ and $R_{t}$ denote the column stabilizer and row stabilizer of $t$, respectively. Let $e_{t}$ be the element of the group algebra $\mathbb{C S y m}(f)$ given by

$$
e_{t}=\frac{1}{\prod h_{i j}} \sum_{\gamma \in C_{t}} \sum_{\sigma \in R_{t}} \operatorname{sgn}(\gamma)(\gamma \sigma),
$$

where $\prod h_{i j}$ is the product of the hook-lengths of $\lambda$. The element $e_{t}$ is called the Young symmetrizer indexed by $t$. It is well known that the set of $e_{t}$ for $t$ an SYT of shape $\lambda$ gives a complete set of orthogonal idempotents for the matrix ring in $\mathbb{C} \operatorname{Sym}(f)$ corresponding to the irreducible $\chi^{\lambda}$.

Let $e_{t}$ be a Young symmetrizer and let $\pi$ be a permutation in $\operatorname{Sym}(f)$. It turns out that the coefficient of $\pi$ in $e_{t}$ is $-1,0$, or 1 . Moreover, there is a combinatorial algorithm to determine this coefficient, which takes no more than $\sum_{j}\left(\alpha_{j}^{2}+\alpha_{j}\right)$ operations, where $\alpha_{j}$ is the number of elements in the $j$ th column of $t$. We will denote this algorithm by $\operatorname{COEF}(\pi, t)$. We know of no reference where this algorithm is discussed explicitly. However, one can construct the algorithm by following the proof of Lemma 1.5.7 on p. 31 of James and Kerber [10].

\section{COMPUTING THE BRAUER ALGEBRA DISCRIMINANTS}

We are now ready to explain how to use Theorem 3.1 to attack the computational problem stated in $\S 2$. In this section, $m$ and $k$ are fixed nonnegative integers and $f=m+2 k$. We will apply Theorem 3.1 with $V$ being $V_{m, k}$, with $Z$ being $Z_{m, k}(x)$, and with $G$ being $\operatorname{Sym}(f) \times \operatorname{Sym}(m)$. Recall from $\S 2$ that there is a natural action of $G$ on $V$, and the matrix $Z$ commutes with this action of $G$. We want to compute the rank and determinant of $Z$. The point of $\S 3$ is that we can derive this information by computing with much smaller matrices $Z_{\lambda, \mu}(x)$ which are indexed by the irreducible representations $\varphi_{\lambda} \otimes \varphi_{\mu}$ of $G$. Theorem 3.1 gives an algorithm for computing the $Z_{\lambda, \mu}(x)$. Our next step in carrying out this algorithm will be to compute the size of $Z_{\lambda, \mu}(x)$, i.e., the multiplicity of $\varphi_{\lambda} \otimes \varphi_{\mu}$ in $V_{m, k}$.

Theorem 4.1. Let $\mu$ and $\lambda$ be partitions of $m$ and $f$, respectively, and let $m(\mu, \lambda)$ denote the multiplicity of $\varphi_{\lambda} \otimes \varphi_{\mu}$ in $V_{m, k}$. Then

$$
m(\mu, \lambda)=\left(\sum_{\substack{\eta \vdash 2 k \\ \eta \text { even }}} g_{\lambda \mu \eta}\right) .
$$

Proof. Let $G$ be $\operatorname{Sym}(f) \times \operatorname{Sym}(m)$, let $H$ be the subgroup

$$
(\operatorname{Sym}(2 k) \times \operatorname{Sym}(m)) \times \operatorname{Sym}(m),
$$


and let $S$ be the subgroup of $H$ given by

$$
S=\left\{(\pi, \sigma, \sigma): \pi \in \mathscr{B}_{2 k}, \sigma \in \operatorname{Sym}(m)\right\}
$$

Here, $\mathscr{B}_{2 k}$ denotes the hyperoctahedral group of $k \times k$ signed permutation matrices, which is considered to be a subgroup of $\operatorname{Sym}(2 k)$ in the usual way.

Note that $G$ acts as a transitive permutation group on the set $B_{m, k}$. So the action of $G$ on $V_{m, k}$ is the induction of the trivial character $\varepsilon$ from the stabilizer of any $\Delta_{0} \in B_{m, k}$ to $G$. Choosing

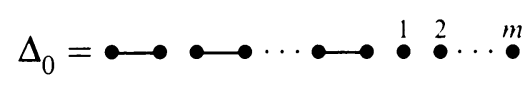

we have that the stabilizer of $\Delta_{0}$ is $S$.

Using a theorem of Littlewood (see Macdonald [14, p. 45, ex. 5]) and some well-known facts about the structure of group algebras, we have

$$
\operatorname{ind}_{S}^{H}(\varepsilon)=\bigoplus_{\substack{\eta \vdash 2 k \\ \eta \text { even }}} \bigoplus_{\mu \vdash m} \varphi_{\eta} \otimes \varphi_{\mu} \otimes \varphi_{\mu} .
$$

By the Littlewood-Richardson rule we have for each $\eta, \mu$

$$
\operatorname{ind}_{H}^{G}\left(\varphi_{\eta} \otimes \varphi_{\mu} \otimes \varphi_{\mu}\right)=\sum_{\lambda \vdash f} g_{\lambda \mu \eta} \varphi_{\lambda} \otimes \varphi_{\mu},
$$

which completes the proof.

At this point we can appreciate how much simplification Theorem 3.1 has to offer us. In the case $m=6, k=3, f=12$, the original matrix $Z_{m, k}(x)$ has dimension on the order of $10,000,000$. Theorem 3.1 says that the matrix splits as a direct sum of matrices $Z_{\lambda, \mu}(x)$ of dimension $m(\mu, \lambda)$, each repeated $f_{\mu} f_{\lambda}$ times. Using Theorem 4.1, one can show that the largest dimension of any of these matrices $Z_{\lambda, \mu}(x)$ is just 15 .

Now fix partitions $\mu \vdash m$ and $\lambda \vdash f$. If $\mu$ is not contained in $\lambda$, then $g_{\lambda \mu \eta}=0$ for all $\eta$, so $m(\mu, \lambda)=0$. Thus, we may assume that $\mu \subseteq \lambda$. We will identify a particular idempotent $e$ in the group algebra of $G$ corresponding to the irreducible representation $\varphi_{\lambda} \otimes \varphi_{\mu}$. To obtain this idempotent, first let $s_{0}$ be the minimal standard Young tableau of shape $\mu$. So:

$$
s_{0}=\left[\begin{array}{cccc}
1 & \cdots & \mu_{1} \\
\left(\mu_{1}+1\right) & \left(\mu_{1}+2\right) & \cdots & \left(\mu_{1}+\mu_{2}\right) \\
& \vdots \\
\left(\mu_{1}+\cdots+\mu_{l-1}+1\right) & \cdots & m
\end{array}\right.
$$

Next, let $t_{0}$ be the standard Young tableau of shape $\lambda$ which agrees with $s_{0}$ on the intersection of $\lambda$ and $\mu$ and which has the minimal filling of $[\lambda / \mu]$ with 
$m+1, \ldots, f$. So, $t_{0}$ looks like

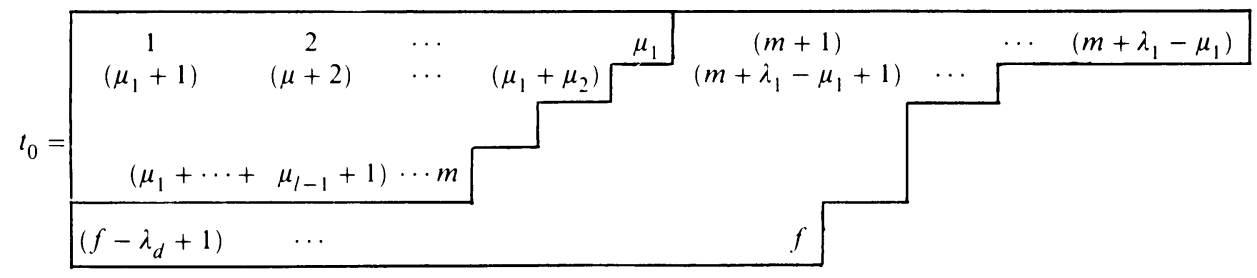

Let $e$ be defined by $e=e_{t_{0}} \times e_{s_{0}}$. We note that $e$ is an idempotent in the group algebra of $G$ corresponding to the irreducible $\varphi_{\lambda} \otimes \varphi_{\mu}$.

Next we need to pick out vectors $v_{1}, \ldots, v_{M}(M=m(\mu, \lambda))$ in $V_{m, k}$ such that $e v_{1}, \ldots, e v_{M}$ are linearly independent. The formula for $m(\mu, \lambda)$ given in Theorem 4.1 suggests how to choose the $v_{i}$. According to the formula, we need one $v_{i}$ for each Littlewood-Richardson filling of $[\lambda / \mu]$ with content $\eta$, where $\eta$ is even. Our actual choice of $v_{i}$ will be put in terms of a certain 1-1 correspondence between 1-factors on $2 k$ points and lattice permutations of length $2 k$ with even content.

In the general case, our proof that the $e v_{i}$ are linearly independent depends on a difficult technical lemma. Rather than obscure the exposition with these details, we will do an interesting special case here and briefly discuss the general case afterwards.

Definition 4.2. We say the pair $(\lambda, \mu)$ is $\mu$-extremal if $[\lambda / \mu]$ has no pair of squares in the same row or the same column.

For the rest of this section we assume that $(\lambda, \mu)$ is $\mu$-extremal. In this case the tableau $t_{0}$ looks like

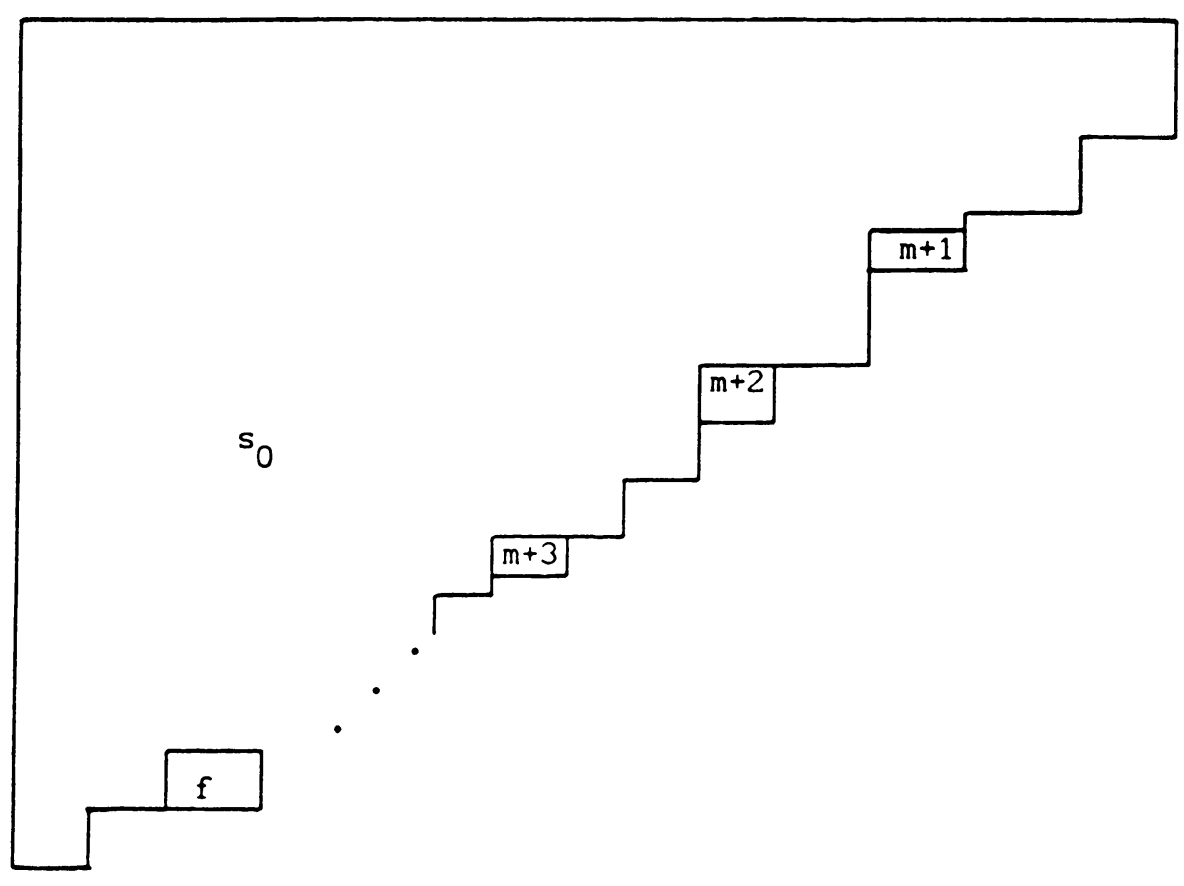


Any lattice permutation of length $2 k$ and shape $\eta$ constitutes a LittlewoodRichardson filling of $[\lambda / \mu]$, so for all $\eta$ we have

$$
g_{i \mu \eta}=f_{\eta} .
$$

Thus,

$$
m(\mu, \lambda)=\sum_{\eta \text { even }} f_{\eta}=1 \cdot 3 \cdots(2 k-1) .
$$

Hence the multiplicity $m(\mu, \lambda)$ equals the number of 1 -factors on $2 k$ points. Note. For any pair $(\lambda, \mu)$ we have $g_{\lambda \mu \eta} \leq f_{\eta}$. So in general, $m(\mu, \lambda) \leq$ $1 \cdot 3 \cdots(2 k-1)$. Equality is achieved if and only if $(\lambda, \mu)$ is $\mu$-extremal.

We want to define a vector $v_{\Delta}$ for each 1 -factor $\Delta$ with $2 k$ points.

Definition 4.3. Let $\Delta$ be a 1 -factor with $2 k$ points. Define the $m, k$ partial 1 -factor $v_{\Delta}$ as follows:

(1) $v_{\Delta}$ has free points $1,2, \ldots, m$. The free point $j$ has label $j$.

(2) For every edge $\{u, v\}$ of $\Delta$ we have the edge $\{m+u, m+v\}$ of $v_{\Delta}$.

The next lemma will not only show that the $e v_{\Delta}$ are linearly independent, it will also greatly streamline our computation. It is this result which is difficult to prove in the case of general pairs $(\lambda, \mu)$.

Lemma 4.4. Let $(\lambda, \mu)$ be $\mu$-extremal and define $t_{0}, s_{0}$ as above. Let $\gamma, \sigma, \gamma^{\prime}$, and $\sigma^{\prime}$ be in $C_{t_{0}}, R_{t_{0}}, C_{s_{0}}$, and $R_{s_{0}}$, respectively $\left(\operatorname{so}, \operatorname{sgn}(\gamma) \operatorname{sgn}\left(\gamma^{\prime}\right)\left(\gamma \sigma, \gamma^{\prime} \sigma^{\prime}\right)\right.$ is one of the terms occurring in the idempotent $\left.e=e_{t_{0}} \times e_{s_{0}}\right)$. Suppose that

$$
\left(\gamma \sigma, \gamma^{\prime} \sigma^{\prime}\right) v_{\Delta}=v_{\widehat{\Delta}},
$$

where $\Delta$ and $\widehat{\Delta}$ are 1-factors. Then

(1) $\Delta=\widehat{\Delta}$,

(2) $\gamma$ and $\sigma$ both fix $t_{0} / s_{0}$ pointwise.

(3) $\gamma$ restricted to $s_{0}$ equals $\gamma^{\prime}$, and $\sigma$ restricted to $s_{0}$ equals $\sigma^{\prime}$. In particular, $\left\{e v_{\Delta}: \Delta\right.$ is a 1-factor with $2 k$ points $\}$ is a basis for $\mathrm{eV} V_{m, k}$.

Proof. Recall that $\gamma^{\prime} \sigma^{\prime}$ acts on an $m, k$ partial 1-factor by changing the labels on the free points by $\left(\gamma^{\prime} \sigma^{\prime}\right)$. Since the free points of both $\Delta$ and $\widehat{\Delta}$ are $1,2, \ldots, m$, it follows that $\gamma \sigma$ preserves the sets $\{1,2, \ldots, m\}$ and $\{m+1, \ldots, f\}$.

In $t_{0}$, each square $m+u(u=1,2, \ldots, 2 k)$ is at the right-hand end of the row containing it and at the bottom of the column containing it. So $\sigma$ moves the point $m+u$ weakly to the left. Since the image of $m+u$ under $\gamma \sigma$ is in the set $\{m+1, \ldots, f\}$, the permutation $\gamma$ must then move $\sigma(m+u)$ down to the bottom of the column it occupies. Thus, $(\gamma \sigma)(m+u)=m+v$, where $v \geq u$. It follows easily that $\gamma \sigma$ fixes the set $\{m+1, \ldots, f\}$ pointwise. So $\Delta=\widehat{\Delta}$, and $\gamma$ and $\sigma$ individually must fix the set $\{m+1, \ldots, f\}$ pointwise.

Next, consider $\gamma \sigma \times \gamma^{\prime} \sigma^{\prime}$ on $s_{0}$. The point $j$ is moved by $\left(\gamma \sigma, \gamma^{\prime} \sigma^{\prime}\right)$ to 
$\gamma \sigma(j)$ and its label is changed to $\left(\gamma^{\prime} \sigma^{\prime}\right) j$. Since $\left(\gamma \sigma, \gamma^{\prime} \sigma^{\prime}\right) v_{\Delta}=v_{\Delta}$, we have

$$
(\gamma \sigma) j=\left(\gamma^{\prime} \sigma^{\prime}\right) j
$$

for all $j$. Thus, $\gamma \sigma=\gamma^{\prime} \sigma^{\prime}$, so $\gamma=\gamma^{\prime}$ and $\sigma=\sigma^{\prime}$, where these last three equalities refer to $\gamma \sigma, \gamma$, and $\sigma$ restricted to the points of $s_{0}$. Suppose $\sum_{\Delta} a_{\Delta} e v_{\Delta}=0$. Then $\sum_{\Delta} a_{\Delta} v_{\Delta}=0$, and so all $a_{\Delta}=0$, as the $v_{\Delta}$ are linearly independent. Thus the set of $e v_{\Delta}$ is a basis, and this finishes the proof.

Lemma 4.4 is the basis for a major simplification of our algorithm. It will show that instead of summing over all the terms of the idempotent $e_{t_{0}} \times e_{s_{0}}$, we can instead work with only those terms which arise from coset representatives of $R_{s_{0}}$ and $C_{s_{0}}$ in $R_{t_{0}}$ and $C_{t_{0}}$. In other words, we can ignore all of the terms in $e_{s_{0}}$ and most of the terms in $e_{t_{0}}$. Before stating the final algorithm, we need notation for these coset representatives and for one of the procedures in the algorithm.

Definition 4.5. Let $\left(a_{i}, b_{i}\right) \quad(i=1,2, \ldots, 2 k)$ be the coordinates of the squares of $[\lambda / \mu]$. For each $i$ define sets $C^{(i)} \subseteq C_{t_{0}}$ and $R^{(i)} \subseteq R_{t_{0}}$ as follows:

(1) $C^{(i)}$ contains the identity permutation as well as the $\left(a_{i}-1\right)$ involutions

$\gamma_{i, j}$ which exchange the elements of $t_{0}$ in squares $\left(a_{i}, b_{i}\right)$ and $\left(j, b_{i}\right)$.

(2) $R^{(i)}$ contains the identity permutation as well as the $\left(b_{i}-1\right)$ involutions

$\sigma_{i, j}$ which exchange the elements of $t_{0}$ in squares $\left(a_{i}, b_{i}\right)$ and $\left(a_{i}, j\right)$.

Let $C$ be the set of all products $\gamma^{(1)} \cdots \gamma^{(2 k)}$, where $\gamma^{(i)} \in C^{(i)}$, and let $R$ be the set of all products $\sigma^{(1)} \cdots \sigma^{(2 k)}$, where $\sigma^{(i)} \in R^{(i)}$. Note that $C$ and $R$ are subsets of $C_{t_{0}}$ and $R_{t_{0}}$ of sizes

$$
|C|=a_{1} a_{2} \cdots a_{2 k}, \quad|R|=b_{1} b_{2} \cdots b_{2 k} .
$$

Our eventual algorithm will compute the $v_{\Delta_{2}}, v_{\Delta_{1}}$ entry in $Z_{\lambda, \mu}(x)$ as a sum of terms of the form $\tau=\left\langle\left(\gamma \sigma, \gamma^{\prime} \sigma^{\prime}\right) v_{\Delta_{2}}, v_{\Delta_{1}}\right\rangle$, where $\gamma \in C, \sigma \in R, \gamma^{\prime} \in C_{s_{0}}$, and $\sigma^{\prime} \in R_{s_{0}}$. For a fixed pair $(\gamma, \sigma) \in C \times R$ there is at most one pair $\left(\gamma^{\prime}, \sigma^{\prime}\right) \in C_{s_{0}} \times R_{s_{0}}$ for which $\tau$ is nonzero. We next write down a method for computing $\Pi=\gamma^{\prime} \sigma^{\prime}$, given $(\gamma, \sigma), v_{\Delta_{2}}$, and $v_{\Delta_{1}}$. In the description below we will assume that the input is $\delta_{1}=\gamma \sigma v_{\Delta_{2}}$ and $\delta_{2}=v_{\Delta_{1}}$.

Definition 4.6. Let $\delta_{1}$ and $\delta_{2}$ be $m, k$ partial 1 -factors. Define an element $\Pi\left(\delta_{1}, \delta_{2}\right)$ in the group algebra $\mathbb{C} \operatorname{Sym}(m)$ according to the following algorithm. For each $i$ in the set $\{1,2, \ldots, m\}$ find the unique path in $\delta_{1} \cup \delta_{2}$ which begins at the free point of $\delta_{1}$ labelled $i$ and ends at some other free point $y$. If $y$ is a free point of $\delta_{1}$, then $\Pi\left(\delta_{1}, \delta_{2}\right)=0$ and the algorithm stops. Otherwise, $y$ is a free point of $\delta_{2}$. Let $\Pi\left(\delta_{1}, \delta_{2}\right)(i)$ be the label on $y$. 
When this algorithm finishes, we will have either $\Pi\left(\delta_{1}, \delta_{2}\right)=0$ or else $\Pi\left(\delta_{1}, \delta_{2}\right) \in \operatorname{Sym}(m)$. For $\delta_{1}, \delta_{2}$ both $m, k$ partial 1 -factors and $r$ a standard Young tableau of size $m$, define $\Gamma_{r}\left(\delta_{1}, \delta_{2}\right)$ by

$$
\Gamma_{r}\left(\delta_{1}, \delta_{2}\right)=\left\{\begin{array}{l}
0 \quad \text { if } \Pi\left(\delta_{1}, \delta_{2}\right)=0, \\
\text { the coefficient of } \Pi\left(\delta_{1}, \delta_{2}\right) \\
\text { in the Young symmetrizer } e_{r} \quad \text { if } \Pi\left(\delta_{1}, \delta_{2}\right) \in \operatorname{Sym}(m) .
\end{array}\right.
$$

Recall that the algorithm COEF discussed in $\S 3$ computes $\Gamma_{r}\left(\delta_{1}, \delta_{2}\right)$ from $\Pi\left(\delta_{1}, \delta_{2}\right)$ in no more than $\sum \alpha_{j}\left(\alpha_{j}+1\right)$ steps, where $\alpha_{j}$ is the length of the $j$ th column of $r$. Also it is easy to see that $\Pi\left(\delta_{1}, \delta_{2}\right)$ together with the number of cycles in $\delta_{1} \cup \delta_{2}$ can be computed in $O(f)$ steps (the actual bound is $f$ or $2 f$ depending on what counts as a step).

Before giving the final algorithm, we prove one additional fact which will be used to increase its efficiency.

Lemma 4.7. Let $\gamma=\gamma^{(1)} \cdots \gamma^{(2 k)}$ be in $C$, and $\sigma=\sigma^{(1)} \cdots \sigma^{(2 k)}$ be in $R$. Suppose that for some $i$, both $\gamma^{(i)}$ and $\sigma^{(i)}$ are not the identity. Then

$$
\Gamma_{s_{0}}\left(\gamma \sigma v_{\Delta_{2}}, v_{\Delta_{1}}\right)=0
$$

Proof. Fix $i$ such that $\gamma^{(i)}=\left(u, b_{i}\right)$ with $u<a_{i}$ and $\sigma^{(i)}=\left(a_{i}, v\right)$ with $v<b_{i}$. Let $\alpha$ and $\beta$ be the labels in the squares $\left(u, b_{i}\right)$ and $\left(a_{i}, v\right)$ of $t_{0}$. The row permutation $\sigma$ moves the label $\beta$ to the corner square $\left(a_{i}, b_{i}\right)$. Then the column permutation $\gamma$ moves the label $\beta$ to the square $\left(u, b_{i}\right)$. So in $\gamma \sigma v_{\Delta_{2}} \cup v_{\Delta_{1}}$ the path beginning at the free point labelled $\beta$ in $\gamma \sigma v_{\Delta_{2}}$ has length 0 and ends at the same point of $v_{\Delta_{1}}$ which is a free point labelled $\alpha$. So,

$$
\Pi\left(\gamma \sigma v_{\Delta_{2}}, v_{\Delta_{1}}\right)(\beta)=\alpha .
$$

But the corner square $\left(a_{i}, b_{i}\right)$ does not exist in $s_{0}$, so $\Pi\left(\gamma \sigma v_{\Delta_{2}}, v_{\Delta_{1}}\right)$ moves $\beta$ from position $\left(a_{i}, v\right)$ to $\left(u, b_{i}\right)$ where $b_{i}>\mu_{a_{i}}$. It is easy to see that such a permutation cannot be written in the form $\hat{\gamma} \hat{\sigma}$ where $\hat{\gamma} \in C_{s_{0}}$ and $\hat{\sigma} \in R_{s_{0}}$. So,

$$
\Gamma_{s_{0}}\left(\gamma \sigma v_{\Delta_{2}}, v_{\Delta_{1}}\right)=0
$$

Let $S$ denote the set of pairs $(\gamma, \sigma)$ with $\gamma=\gamma^{(1)} \cdots \gamma^{(2 k)} \in C$ and $\sigma=$ $\sigma^{(1)} \cdots \sigma^{(2 k)} \in R$ such that $\sigma^{(i)}$ is the identity whenever $\gamma^{(i)}$ is not the identity. Note that the size of $S$ is

$$
|S|=\prod_{i=1}^{2 k}\left(a_{i}+b_{i}-1\right)
$$

Theorem 4.8. The following algorithm computes the $\Delta_{i}, \Delta_{j}$ entry in $Z_{\lambda, \mu}(x)$. Algorithm. For each pair $(\gamma, \sigma) \in S$

(1) Compute $\Gamma_{s_{0}}\left(\gamma \sigma v_{\Delta_{i}}, v_{\Delta_{i}}\right)$.

(2) Compute the number of cycles $N$ in $\gamma \sigma v_{\Delta_{j}} \cup v_{\Delta_{i}}$.

(3) Add $\operatorname{sgn}(\gamma) \Gamma_{s_{0}}\left(\gamma \sigma v_{\Delta_{j}}, v_{\Delta_{i}}\right) x^{N}$ to the current value of $Z_{\lambda, \mu}(x)$. 
Before proving that the algorithm in Theorem 4.8 works, we make some remarks on its efficiency. Consider the case $\lambda=654321$ and $\mu=54321$. The size of our original matrix $Z_{m, k}(x)$ is a whopping (21)!/48. The submatrix $Z_{\lambda, \mu}(x)$ that we wish to extract is $15 \times 15$. The six squares of $[\gamma / \mu]$ have coordinates $(1,6),(2,5),(3,4),(4,3),(5,2)$, and $(6,1)$, so the size of $S$ is $6^{6}$. Thus each entry of $Z_{\lambda, \mu}(x)$ is computed with $6^{6}$ passes through the main loop of the algorithm in Theorem 4.8. In practice, this matrix $Z_{\lambda, \mu}(x)$ was computed in about one hour of CPU time on a CRAY-2.

In general, we must perform the main loop in the above algorithm $\prod\left(a_{i}+b_{i}-1\right)$ times. This main loop is carried out in $O\left(f+\sum \alpha_{j}\left(\alpha_{j}+1\right)\right)$ steps. So Theorem 4.8 gives a method to compute each entry of $Z_{\lambda, \mu}(x)$ in

$$
o\left\{\prod\left(a_{i}+b_{i}-1\right)\left(f+\sum \alpha_{j}\left(\alpha_{j}+1\right)\right)\right\}
$$

steps. In practice, this is efficient enough to work out a large number of cases, as the data in the next section will indicate.

We now sketch a proof of Theorem 4.8. Let $e=c_{s_{0}} r_{s_{0}}$ be the Young symmetrizer indexed by $s_{0}$. According to Theorem 3.1, the matrix $Z_{m, k}(x)$ preserves the subspace $\left\langle e v_{\Delta}: \Delta\right.$ is a 1 -factor of size $\left.2 k\right\rangle$. By Lemma 4.4 the coefficient of $v_{\Delta_{i}}$ in $e v_{\Delta_{j}}$ is 0 for $i \neq j$ and is $\left|R_{s_{0}}\right|\left|C_{s_{0}}\right|$ for $i=j$. So the $i, j$ entry of $Z_{\lambda, \mu}(x)$ is $\left(1 / \mid R_{s_{0}} \| C_{s_{0}}\right)$ times the coefficient of $v_{\Delta_{i}}$ in $Z_{\lambda, \mu}(x)\left(e v_{\Delta_{j}}\right)$. Thus,

$$
\begin{aligned}
\left(Z_{\lambda, \mu}(x)\right)_{\Delta_{i}, \Delta_{j}} & =\frac{1}{\left|R_{s_{0}}\right|\left|C_{s_{0}}\right|}\left\langle e v_{\Delta_{j}}, v_{\Delta_{i}}\right\rangle \\
& =\frac{1}{\left|R_{s_{0}}\right|\left|C_{s_{0}}\right|} \sum_{\substack{\gamma \in C_{t_{0}} \\
\sigma \in R_{t_{0}}}} \operatorname{sgn}(\gamma) \sum_{\substack{\gamma^{\prime} \in C_{s_{0}} \\
\sigma^{\prime} \in R_{s_{0}}}} \operatorname{sgn}\left(\gamma^{\prime}\right)\left\langle\left(\gamma \sigma, \gamma^{\prime} \sigma^{\prime}\right) v_{\Delta_{j}}, v_{\Delta_{i}}\right\rangle \\
& =\sum_{\gamma \in C} \sum_{\sigma \in R} \operatorname{sgn}(\gamma) \Gamma_{s_{0}}\left(\gamma \sigma v_{\Delta_{j}}, v_{\Delta_{i}}\right)\left\langle\gamma \sigma v_{\Delta_{j}}, v_{\Delta_{i}}\right\rangle .
\end{aligned}
$$

The last equality follows easily from the definition of $\Gamma_{s_{0}}$. Now by Lemma 4.7 we have

$$
\left(Z_{\lambda, \mu}(x)\right)_{\Delta_{i}, \Delta_{j}}=\sum_{(\gamma, \sigma) \in S} \operatorname{sgn}(\gamma) \Gamma_{s_{0}}\left(\gamma \sigma v_{\Delta_{j}}, v_{\Delta_{i}}\right)\left\langle\gamma \sigma v_{\Delta_{j}}, v_{\Delta_{i}}\right\rangle,
$$

which completes the proof.

We end this section with an example of how Theorem 4.8 can be used to compute an arbitrary entry of $Z_{\lambda, \mu}(x)$. According to Theorem 3.1, there exists a matrix similar to $Z_{2,1}(x)$ which breaks up as a direct sum. One of the summands corresponds to the irreducible representation $\varphi_{31} \otimes \varphi_{2}$ of $\operatorname{Sym}(4) \times$ $\operatorname{Sym}(2)$, and this summand is itself a direct sum of $\left(f_{31} f_{2}\right)=3$ one by one matrices $Z_{31,2}(x)$. Theorem 4.8 tells us how to compute the matrix $Z_{31,2}(x)$ directly. 
According to Theorem 4.8, the matrix $Z_{31.2}(x)$ is a sum over all pairs $(\gamma, \sigma)$ in $S$. The tableaux $t_{0}$ and $s_{0}$ are

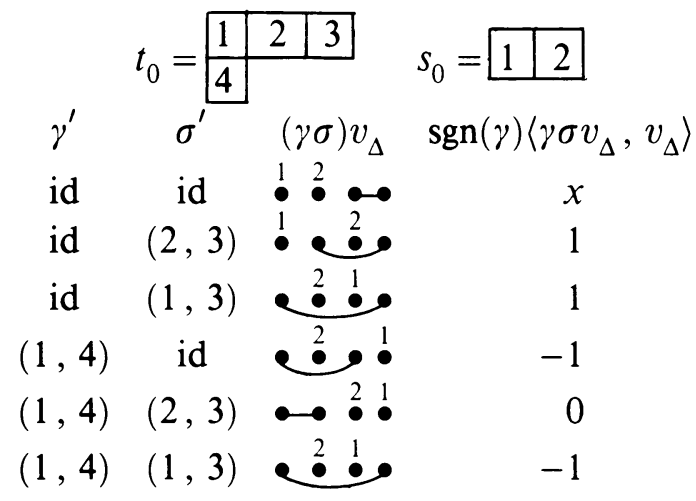

Summing the right-hand column gives $Z_{31,2}(x)=[x]$.

Some readers may be familiar with the "Meataxe" programs developed at Cambridge by R. Parker. These are exceptionally good algorithms for finding subspaces invariant under a linear transformation $T$. The algorithms just described perform the same function on the matrix $Z_{m, k}(x)$ as the Meataxe. However, the Meataxe is not suitable for use in this situation because of the enormous size of the matrices $Z_{m, k}(x)$. The Meataxe uses a different approach than the algorithms developed in this paper because it is designed for use in a very general situation. Since the Meataxe is powerful enough to be applied in a general setting, it is limited in the size of the matrix that it can "chop up". Our algorithms will handle a much larger size matrix, but they are highly customized to the specific situation.

The algorithm we just described computes $Z_{\lambda, \mu}(x)$ in the case that $[\lambda / \mu]$ has squares in distinct rows and columns. In the case where $[\lambda / \mu]$ does not have that property, most of the algorithm goes exactly as before. The important difference comes in how we choose vectors $v_{1}, \ldots, v_{M}(M=m(\mu, \lambda))$ so that $e v_{1}, \ldots, e v_{M}$ are linearly independent. We will now describe how to make that choice. The choice will use Schensted's correspondence and the following interesting property of Schensted's correspondence, originally due to Knuth (see [15]).

Theorem 4.9. Schensted column insertion gives a 1-1 correspondence between fuxed-point free involutions in $\operatorname{Sym}(2 k)$ and standard Young tableaux whose shape has even column lengths.

There is an obvious bijection between fixed-point free involutions in $\operatorname{Sym}(2 k)$ and 1 -factors on $2 k$ points. So the above theorem gives a bijection between standard Young tableaux whose shape is a column-even partition of $2 k$ and 1 -factors on $2 k$ points.

Now let $(\lambda, \mu)$ be an arbitrary pair of partitions of $f$ and $m$. Recall that 
the multiplicity $m(\mu, \lambda)$ is given by

$$
m(\mu, \lambda)=\sum_{\substack{\eta \vdash 2 k \\ \eta \text { even }}} g_{\lambda \mu \eta}
$$

So we need to come up with one vector $v_{\phi}$ for every Littlewood-Richardson filling $\phi$ of $[\lambda / \mu]$ having content $\eta$ an even partition of $2 k$. The procedure to find $v_{\phi}$ is as follows.

Step 1: Read from $\phi$ the corresponding lattice permutation $s_{1}, s_{2}, \ldots$, $s_{2 k}$ of content $\eta$.

Step 2: From $s_{1}, s_{2}, \ldots, s_{2 k}$ produce the standard Young tableau $t_{\phi}$ which has $i$ in column $s_{i}$ for $i=1,2, \ldots, 2 k$.

Step 3: Use the bijection given by Schensted's correspondence to get a 1 -factor $\delta_{\phi}$ from $t_{\phi}$.

Step 4: Define $v_{\phi}$ to be the $m, k$ partial 1-factor with free points $1,2, \ldots, m$, labelled $1,2, \ldots, m$, respectively, and with an edge from $m+i$ to $m+j$ for each edge $\{i, j\}$ of $\delta_{\phi}$.

Theorem 4.10. For each $L-R$ filling $\phi$ of $[\lambda / \mu]$ having even content, let $v_{\phi}$ be the vector defined by the procedure above. Then the set of $e v_{\phi}$ is linearly independent.

The vectors $v_{\phi}$ have many remarkable combinatorial properties owing to the fact that $\phi$ is a Littlewood-Richardson filling. The proof of Theorem 4.10 is based on a careful analysis of these properties and is long and detailed. It produces an abundance of combinatorial information, but has little bearing on the general computational method being described in this paper. For the sake of brevity we leave it out.

\section{RESULTS OF THE COMPUTATIONS}

We implemented the algorithm outlined in $\S 4$ on the CRAY-1 and CRAY-2 supercomputers at the University of Minnesota. The code was written to take advantage of some features of the machines' architectures. The authors wish to thank the National Science Foundation for supplying supercomputer time.

The first table lists the roots of the determinant of $Z_{\lambda, \mu}(x)$ for all pairs $(\lambda, \mu)$ where $\lambda$ is a partition of 8 or less. Table 2 gives this same information for certain larger $(\lambda, \mu)$ which are particularly interesting. Lastly, Table 3 gives the eigenvalues of the matrices $Z_{\lambda, \mu}(x)$ for some small cases.

Some of the roots in this table have stars and some of the rows have check marks in the last two columns which are headed " $\eta$-extremal" and " $\mu$-extremal". These bits of information refer to theorems and conjectures which will be discussed in $\S 6$. 
TABLE 1

\begin{tabular}{|c|c|c|c|c|c|}
\hline & & & & & emal \\
\hline$\lambda$ & $\mu$ & $k\left(\sum_{\eta} g_{\lambda \mu \eta}\right)$ & Roots of $\operatorname{det}\left(Z_{\dot{\lambda}, \mu}(x)\right)$ & $\bar{\eta}$ & $\bar{\mu}$ \\
\hline 2 & $\phi$ & 1 & 0 & $\mathrm{X}$ & \\
\hline 3 & 1 & 1 & -2 & $\mathrm{X}$ & \\
\hline 21 & 1 & 1 & 1 & $\mathrm{X}$ & $\mathrm{X}$ \\
\hline 4 & $\phi$ & 2 & $0,-2$ & $\mathrm{X}$ & \\
\hline 4 & 2 & 1 & $-4^{*}$ & & \\
\hline 31 & 2 & 1 & 0 & $\mathrm{X}$ & $\mathrm{X}$ \\
\hline 31 & $1^{2}$ & 1 & -2 & $\mathrm{X}$ & \\
\hline $2^{2}$ & $\phi$ & 2 & 0,1 & $\mathrm{X}$ & \\
\hline $2^{2}$ & 2 & 1 & 2 & & \\
\hline $21^{2}$ & $1^{2}$ & 1 & 2 & & $\mathrm{X}$ \\
\hline 5 & 1 & 2 & $-2,-4$ & $\mathrm{X}$ & \\
\hline 5 & 3 & 1 & -6 & & \\
\hline 41 & 1 & 2 & $1,-2$ & $\mathrm{X}$ & \\
\hline 41 & 3 & 1 & -1 & & $\mathrm{X}$ \\
\hline 41 & 21 & 1 & $-4^{*}$ & & \\
\hline 32 & 1 & 2 & $1,-2$ & $\mathrm{X}$ & \\
\hline 32 & 3 & 1 & 2 & & \\
\hline 32 & 21 & 1 & -1 & & $\mathrm{X}$ \\
\hline $31^{2}$ & 21 & 1 & 1 & & $\mathrm{X}$ \\
\hline $31^{2}$ & $1^{3}$ & 1 & -2 & & $\mathrm{X}$ \\
\hline $2^{2} 1$ & 1 & 2 & 1,2 & $\mathrm{X}$ & \\
\hline $2^{2} 1$ & 21 & 1 & 3 & & $\mathrm{X}$ \\
\hline $21^{3}$ & $1^{3}$ & 1 & 3 & $\mathrm{X}$ & $\mathrm{X}$ \\
\hline 6 & $\phi$ & 3 & $0,-2,-4$ & $\mathrm{X}$ & \\
\hline 6 & 2 & 2 & $-4,-6^{*}$ & & \\
\hline 6 & 4 & 1 & $-8^{*}$ & & \\
\hline 51 & 2 & 2 & $0,-4$ & $\mathrm{X}$ & \\
\hline 51 & $1^{2}$ & 2 & $-2,-4$ & & \\
\hline 51 & 4 & 1 & -2 & & $\mathrm{X}$ \\
\hline 51 & 31 & 1 & $-6^{*}$ & & \\
\hline 42 & $\phi$ & 3 & $0,-2,1$ & $\mathrm{X}$ & \\
\hline 42 & 2 & 4 & $0,2,-1,-4$ & & \\
\hline 42 & 4 & 1 & 2 & & \\
\hline 42 & 31 & 1 & -2 & & $\mathrm{X}$ \\
\hline 42 & $2^{2}$ & 1 & -4 & & \\
\hline $41^{2}$ & $1^{2}$ & 2 & $2,-2$ & $\mathrm{X}$ & \\
\hline $41^{2}$ & 31 & 1 & 0 & & $\mathrm{X}$ \\
\hline $41^{2}$ & $21^{2}$ & 1 & -4 & & \\
\hline $3^{2}$ & $1^{2}$ & 2 & $-1,-2$ & $\mathrm{X}$ & \\
\hline $3^{2}$ & 31 & 1 & 0 & & \\
\hline 321 & $1^{2}$ & 2 & $2,-2$ & $\mathrm{X}$ & \\
\hline 321 & 2 & 2 & 0,2 & $\mathrm{X}$ & \\
\hline 321 & 31 & 1 & 3 & & $\mathrm{X}$ \\
\hline 321 & $2^{2}$ & 1 & 1 & & $\mathrm{X}$ \\
\hline 321 & $21^{2}$ & 1 & -1 & & $\mathrm{X}$ \\
\hline $31^{3}$ & $21^{2}$ & 1 & 2 & & $\mathrm{X}$ \\
\hline $31^{3}$ & $1^{4}$ & 1 & -2 & & \\
\hline $2^{3}$ & $\phi$ & 3 & $0,1,2$ & $\mathrm{X}$ & \\
\hline $2^{3}$ & 2 & 2 & 2,3 & & \\
\hline
\end{tabular}


TABLE 1 (continued)

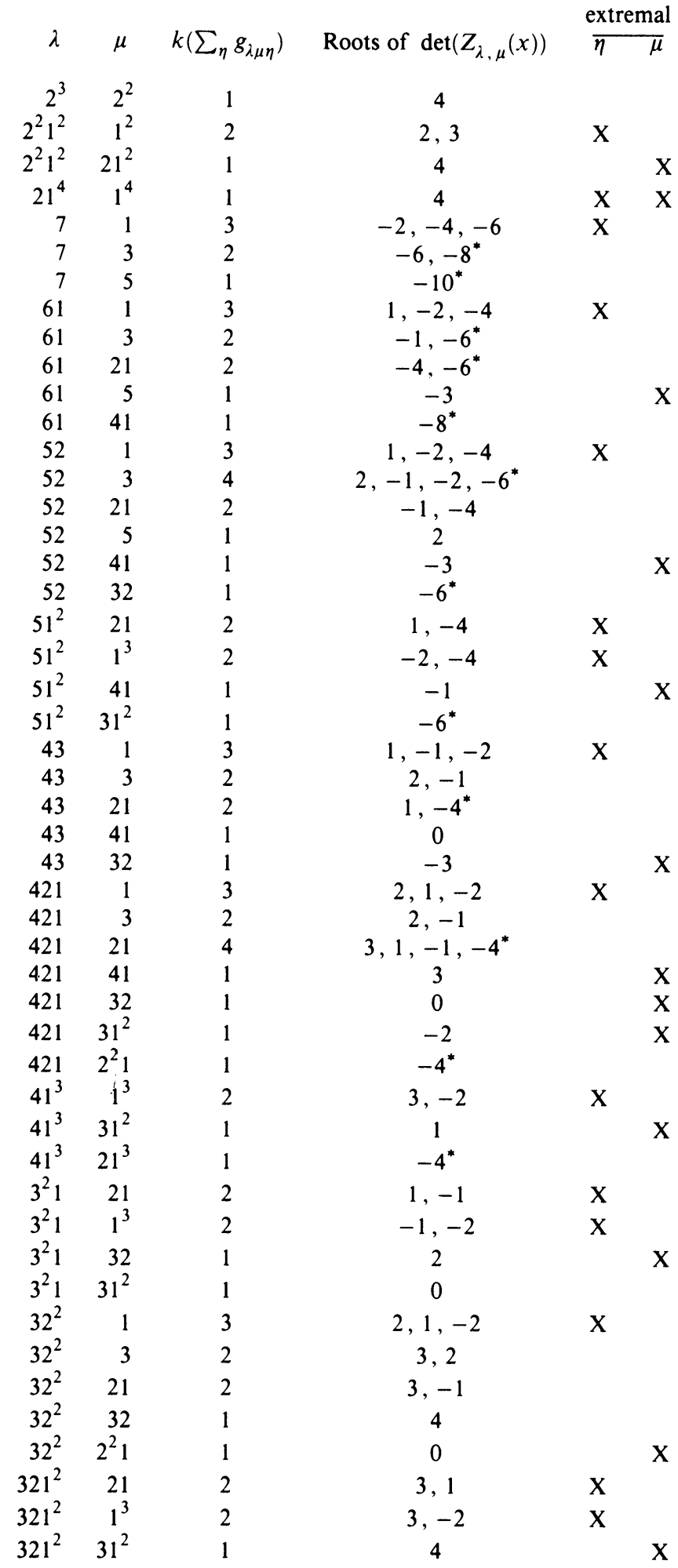




\section{TABLE 1 (continued)}

$$
\lambda \quad \mu \quad k\left(\sum_{\eta} g_{\lambda \mu \eta}\right) \quad \text { Roots of } \operatorname{det}\left(Z_{\lambda, \mu}(x)\right) \quad \frac{\text { extremal }}{\eta \mu}
$$

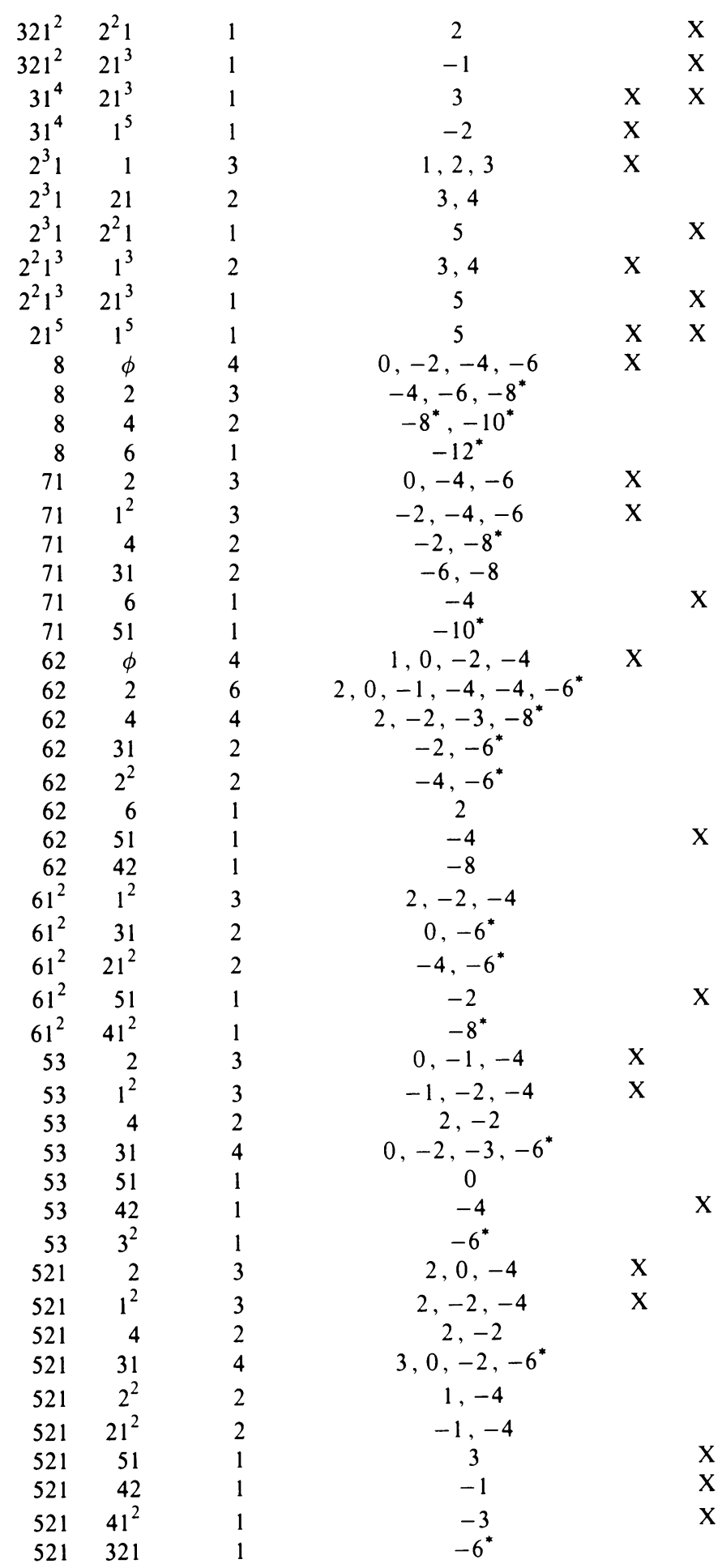


TABLE 1 (continued)

\begin{tabular}{|c|c|c|c|c|c|}
\hline & & & & extr & mal \\
\hline$\lambda$ & $\mu$ & $k\left(\sum_{\eta} g_{\lambda \mu \eta}\right)$ & Roots of $\operatorname{det}\left(Z_{\lambda, \mu}(x)\right)$ & $\bar{\eta}$ & $\bar{\mu}$ \\
\hline $51^{3}$ & $21^{2}$ & 2 & $2,-4$ & & \\
\hline $51^{3}$ & $1^{4}$ & 2 & $-2,-4$ & & \\
\hline $51^{3}$ & $41^{2}$ & 1 & 0 & & $\mathrm{X}$ \\
\hline $51^{3}$ & $31^{3}$ & 1 & $-6^{*}$ & & \\
\hline $4^{2}$ & $\phi$ & 4 & $1,0,-1,-2$ & $\mathrm{X}$ & \\
\hline $4^{2}$ & 2 & 3 & $2,-1,-4^{*}$ & & \\
\hline $4^{2}$ & 4 & 2 & 2,0 & & \\
\hline $4^{2}$ & $2^{2}$ & 2 & $-3,-4^{*}$ & & \\
\hline $4^{2}$ & 42 & 1 & -2 & & \\
\hline 431 & 2 & 3 & $2,0,-1$ & $\mathrm{X}$ & \\
\hline 431 & $1^{2}$ & 3 & $2,-1,-2$ & $\mathrm{x}$ & \\
\hline 431 & 31 & 4 & $3,0,0,2$ & & \\
\hline 431 & $2^{2}$ & 2 & $1,-4^{*}$ & & \\
\hline 431 & $21^{2}$ & 2 & $-1,-4^{*}$ & & \\
\hline 431 & 42 & 1 & 2 & & $\mathrm{X}$ \\
\hline 431 & $3^{2}$ & 1 & 0 & & $\mathrm{X}$ \\
\hline 431 & $41^{2}$ & 1 & 0 & & \\
\hline 431 & 321 & 1 & -3 & & $\mathrm{X}$ \\
\hline $42^{2}$ & $\phi$ & 4 & $2,1,0,-2$ & $\mathrm{X}$ & \\
\hline $42^{2}$ & 2 & 6 & $3,2,2,0,-1,-4^{*}$ & & \\
\hline $42^{2}$ & 4 & 2 & 3,2 & & \\
\hline $42^{2}$ & 31 & 2 & $3,-2$ & & \\
\hline $42^{2}$ & $2^{2}$ & 4 & $4,1,0,-4^{*}$ & & \\
\hline $42^{2}$ & 42 & 1 & 4 & & \\
\hline $42^{2}$ & 321 & 1 & -1 & & $\mathrm{X}$ \\
\hline $42^{2}$ & $2^{3}$ & 1 & $-4^{*}$ & & \\
\hline $421^{2}$ & $1^{2}$ & 3 & $3,2,-2$ & & \\
\hline $421^{2}$ & 31 & 2 & 3,0 & & \\
\hline $421^{2}$ & $21^{2}$ & 4 & $4,2,-1,-4^{*}$ & & \\
\hline $421^{2}$ & $41^{2}$ & 1 & 4 & & $\mathrm{X}$ \\
\hline $421^{2}$ & 321 & 1 & 1 & & $\mathrm{x}$ \\
\hline $421^{2}$ & $31^{3}$ & 1 & -2 & & $\mathrm{X}$ \\
\hline $421^{2}$ & $2^{2} 1^{2}$ & 1 & $-4^{*}$ & & \\
\hline $41^{4}$ & $1^{4}$ & 2 & $4,-2$ & & \\
\hline $41^{4}$ & $31^{3}$ & 1 & 2 & & $\mathrm{X}$ \\
\hline $41^{4}$ & $21^{4}$ & 1 & $-4^{*}$ & & \\
\hline $3^{2} 2$ & $1^{2}$ & 3 & $2,-1,-2$ & & \\
\hline $3^{2} 2$ & 31 & 2 & 3,0 & & \\
\hline $3^{2} 2$ & $21^{2}$ & 2 & $0,-1$ & & \\
\hline $3^{2} 2$ & $3^{2}$ & 1 & 4 & & \\
\hline $3^{2} 2$ & 321 & 1 & 1 & & $\mathrm{X}$ \\
\hline $3^{2} 1^{2}$ & $2^{2}$ & 2 & 2,1 & & \\
\hline $3^{2} 1^{2}$ & $21^{2}$ & 2 & $2,-1$ & & \\
\hline $3^{2} 1^{2}$ & $1^{4}$ & 2 & $-1,-2$ & & \\
\hline $3^{2} 1^{2}$ & 321 & 1 & 3 & & $\mathrm{X}$ \\
\hline $3^{2} 1^{2}$ & $31^{3}$ & 1 & 0 & & \\
\hline $32^{2} 1$ & 2 & 3 & $3,2,0$ & $\mathrm{X}$ & \\
\hline
\end{tabular}


TABLE 1 (continued)

\begin{tabular}{|c|c|c|c|c|c|}
\hline & & & & ext & \\
\hline$\lambda$ & $\mu$ & $k\left(\sum_{\eta} g_{\lambda \mu \eta}\right)$ & Roots of $\operatorname{det}\left(Z_{\lambda, \mu}(x)\right)$ & $\bar{\eta}$ & $\bar{\mu}$ \\
\hline $32^{2} 1$ & $1^{2}$ & 3 & $3,2,-2$ & $X$ & \\
\hline $32^{2} 1$ & 31 & 2 & 4,3 & & \\
\hline $32^{2} 1$ & $2^{2}$ & 2 & 4,1 & & \\
\hline $32^{2} 1$ & $21^{2}$ & 2 & $4,-1$ & & \\
\hline $32^{2} 1$ & 321 & 1 & 5 & & $\mathrm{X}$ \\
\hline $32^{2} 1$ & $2^{3}$ & 1 & 2 & & $\mathrm{x}$ \\
\hline $32^{2} 1$ & $2^{2} 1^{2}$ & 1 & 0 & & $\mathrm{X}$ \\
\hline $321^{3}$ & $21^{3}$ & 2 & 4,2 & & \\
\hline $321^{3}$ & $1^{4}$ & 2 & $4,-2$ & & \\
\hline $321^{3}$ & $31^{3}$ & 1 & 5 & & $X$ \\
\hline $321^{3}$ & $2^{2} 1^{2}$ & 1 & 3 & & $\mathrm{X}$ \\
\hline $321^{3}$ & $21^{4}$ & 1 & -1 & & $\mathrm{X}$ \\
\hline $31^{5}$ & $21^{4}$ & 1 & 4 & & $\mathrm{X}$ \\
\hline $31^{5}$ & $1^{6}$ & 1 & -2 & & \\
\hline $2^{4}$ & $\phi$ & 4 & $3,2,1,0$ & $X$ & \\
\hline $2^{4}$ & 2 & 3 & $4,3,2$ & & \\
\hline $2^{4}$ & $2^{2}$ & 2 & 5,4 & & \\
\hline $2^{4}$ & $2^{3}$ & 1 & 6 & & \\
\hline $2^{3} 1^{2}$ & $1^{2}$ & 3 & $4,3,2$ & & \\
\hline $2^{3} 1^{2}$ & $21^{2}$ & 2 & 5,4 & & \\
\hline $2^{3} 1^{2}$ & $2^{2} 1^{2}$ & 1 & 6 & & $X$ \\
\hline $2^{2} 1^{4}$ & $1^{4}$ & 2 & 5,4 & & \\
\hline $2^{2} 1^{4}$ & $21^{4}$ & 1 & 6 & & $X$ \\
\hline $21^{6}$ & $1^{6}$ & 1 & 6 & & $X$ \\
\hline
\end{tabular}

TABLE 2. This table contains the roots of $\operatorname{det}\left(Z_{\lambda, \mu}(x)\right)$ for some larger values of $\lambda$ and $\mu$.

\begin{tabular}{|c|c|c|}
\hline$\lambda$ & $\mu$ & Roots of $\operatorname{det}\left(Z_{\lambda, \mu}(x)\right)$ \\
\hline 531 & 3 & $2,-1,-2$ \\
\hline 531 & 21 & $1,-1,-4$ \\
\hline 531 & $1^{3}$ & $-1,-2,-4$ \\
\hline 531 & 41 & $3,0,-1,-3$ \\
\hline 10 & $\phi$ & $0,-2,-4,-6,-8$ \\
\hline 10 & 2 & $-4,-6,-8,-10$ \\
\hline 10 & 4 & $-8,-10,-12$ \\
\hline 10 & 6 & $-12,-14$ \\
\hline 10 & 8 & -16 \\
\hline $5^{2}$ & 1,1 & $-1,-2,-3,-4$ \\
\hline 642 & 2 & $3,2,2,2,0,0,0,-1,-1,-1,-3,-4,-4,-4,-6$ \\
\hline 642 & 4 & $3,3,2,2,2,0,0,-2,-2,-3,-3,-8$ \\
\hline 642 & $2^{2}$ & $4,1,1,0,0,-3,-3,-4,-4,-4,-6,-6$ \\
\hline 4321 & 321 & \\
\hline 5321 & 421 & \\
\hline 6321 & 521 & $\begin{array}{l}\text { These six are } \mu \text {-extremal. The roots of } \\
\operatorname{det}\left(Z_{\lambda, \mu}(x)\right) \text { are exactly the roots predicted }\end{array}$ \\
\hline 54321 & 4321 & by the $\mu$-extremal conjecture, Conjecture 6.7 . \\
\hline 64321 & 5321 & \\
\hline 543211 & 43211 & \\
\hline
\end{tabular}




\section{RESULTS AND CONJECTURES}

In this section we state what is known and what is conjectured about the discriminants of the Brauer algebras. The conjectures are based on the computational evidence that we have been able to gather. These conjectures are verified in all reasonably small cases and represent a number of very interesting open problems of an algebraic/combinatorial nature.

A. An important result. Numerical evidence together with two previous results (Theorems 6.6 and 6.10 below) led the authors to conjecture that the roots of $\operatorname{det}\left(Z_{\lambda, \mu}(x)\right)$ are in $\mathbb{Z}$ for all $\lambda$ and $\mu$. In algebraic terms, this is equivalent to the conjecture that the Brauer centralizer algebras are semisimple except possibly at integer values of the multiplication constant $x$.

Theorem 6.1 (Wenzl [18]). If $x \notin \mathbb{Z}$, then the algebra $A_{f}^{(x)}$ is semisimple.

Wenzl actually proves that the centralizer algebra of left multiplication by $A_{f-1}^{(x)}$ in $\operatorname{End}\left(A_{f}^{(x)}\right)$ is isomorphic to $A_{f+1}^{(x)}(1)$ when $x \notin \mathbb{Z}$. It follows immediately that $A_{f+1}^{(x)}$ is semisimple (for $x \notin \mathbb{Z}$ ) by induction on $f$. His proof relies on a construction due to Birman and Wenzl (see [1, §3]), which is in turn a generalization of a construction due to Vaughan Jones (see [11]).

In view of Theorem 6.1, it seems all the more plausible that there is a combinatorial description of the roots of $\operatorname{det}\left(Z_{\lambda, \mu}(x)\right)$. It is known that the roots of $\operatorname{det}\left(Z_{\lambda, \mu}(x)\right)$ cannot be too large in absolute value. The first such result is due to Brauer.

Theorem 6.2 (R. Brauer [3]). If $\lambda$ is a partition of $f$ and $z$ is a nonnegative integral root of $\operatorname{det}\left(Z_{\lambda, \mu}(x)\right)$, then $z<f$. If $z$ is a negative, even integral root of $\operatorname{det}\left(Z_{\lambda, \mu}(x)\right)$, then $|z|<2 f$.

Proof. In [3], Brauer showed that the homomorphism $\varphi_{f}^{(n)}$ from $\mathscr{A}_{f}^{(n)}$ onto End $_{\mathrm{O}(n, \mathbb{R})}\left(T^{f} \mathbb{R}^{n}\right)$ is an isomorphism if $n$ is a positive integer greater than or equal to $f$. Since $\operatorname{End}_{\mathrm{O}(n, \mathbb{R})}\left(T^{f} \mathbb{R}^{n}\right)$ is a semisimple ring, it follows that $\mathscr{A}_{f}^{(n)}$ is semisimple, so the discriminants $\operatorname{det}\left(Z_{\lambda, \mu}(n)\right)$ must be nonzero.

Similarly, Brauer showed that the maps $\psi_{f}^{(2 n)}$ from $\mathscr{B}_{f}^{(2 n)}$ onto

$$
\operatorname{End}_{\operatorname{Sp}(2 n, \mathbb{R})}\left(T^{f} \mathbb{R}^{2 n}\right)
$$

are isomorphisms if $n \geq f$ (so $2 n \geq 2 f$ ). Hence, the discriminants of $\mathscr{B}_{f}^{(2 n)}$ are nonzero if $2 n \geq 2 f$. Since $\mathscr{B}_{f}^{(x)} \cong \mathscr{A}_{f}^{(-x)}$, the result follows.

It is clear from the data in $\S 5$ what bound should hold on the roots of the Brauer algebra discriminants.

Conjecture 6.3. Let $z$ be an integral root of $\operatorname{det}\left(Z_{i, \mu}(x)\right)$.

(1) if $z$ is nonnegative, then $z \leq\left(f-\lambda_{1}\right)$;

(2) if $z$ is negative, then $|z| \leq 2\left(\lambda_{1}-2\right)$. 
B. The extremal cases. When considering pairs $(\lambda, \mu)$, there are two natural extremal cases that arise. One is the case discussed in $\S 4$, which we called $\mu$ extremal. In this case, the squares of $[\lambda / \mu]$ are in distinct rows and columns, so that for any even partition $\eta$ we have

$$
g_{\lambda \mu \eta}=f_{\eta} .
$$

The other extremal case is what we call $\eta$-extremal.

Definition 6.4. The pair $(\lambda, \mu)$ is called $\eta$-extremal if the partition $\lambda$ has exactly $m=|\mu|$ rows of odd length, and these rows have distinct lengths.

The $\eta$-extremal case is in some sense opposite to the $\mu$-extremal case. If the pair $(\lambda, \mu)$ is $\eta$-extremal, then there is a unique even shape $\eta$ for which $g_{\lambda \mu \eta}$ is nonzero. This $\eta$ is obtained by removing one square from each row of $\lambda$ which has odd length. For this particular $\eta$ we have

$$
g_{\lambda \mu \eta}=f_{\mu} .
$$

In the $\eta$-extremal case we know exactly what the eigenvalues of $Z_{\lambda, \mu}(x)$ are. To state this result, we need an expression due to El Samra and King for the dimension of an irreducible $\operatorname{Sp}(2 n, \mathbb{R})$ module.

For any partition $\mu$ and large enough values of $n$, there is an irreducible $\operatorname{Sp}(2 n, \mathbb{R})$-module indexed by $\mu$, which we will denote by $V_{2 n}^{\mu}$.

Theorem 6.5 (El Samra and King [5]). There is a polynomial $d_{\mu}(x)$ with integer roots such that whenever $n$ is large enough for $\mu$ to index an irreducible $\operatorname{Sp}(2 n, \mathbb{R})$-module $V_{2 n}^{\mu}$, then

$$
\operatorname{dim}\left(V_{2 n}^{\mu}\right)=\frac{d_{\mu}(2 n)}{\left(\prod h_{i j}\right)} .
$$

Here, $\left(\prod h_{i j}\right)$ denotes the product of the hook-lengths of $\mu$.

King shows that the polynomial $d_{\mu}(x)$ is monic of degree $m=|\mu|$. Because of this, one can write $d_{\mu}(x)$ in the form $\prod_{y \in \mu}\left(x+r_{y}\right)$, where the product is over all squares $y$ in $[\mu]$, and $r_{y}$ is an integer root corresponding to the square $y$. King showed that if $y$ has coordinates $(i, j)$, then

$$
r_{y}= \begin{cases}i+j-\lambda_{i}-\lambda_{j}-2 & \text { if } i<j \\ \lambda_{i}+\lambda_{j}-i-j & \text { if } i \geq j\end{cases}
$$

The following theorem completely determines the roots of the Brauer algebra discriminants in the $\eta$-extremal case. This result was originally conjectured by R. P. Stanley (based on computational evidence).

Theorem 6.6 (Hanlon and Wales [8]). Suppose the pair $(\lambda, \mu)$ is $\eta$-extremal. Then $Z_{\lambda, \mu}(x)$ is an $f_{\mu} \times f_{\mu}$ scalar matrix $h_{\lambda, \mu}(x) I$. The value of the scalar is

$$
h_{\lambda, \mu}(x)=g_{\lambda}(x) / d_{\mu}(x),
$$

where $g_{\lambda}(x)=\prod_{(i, 2 j-1) \in[\lambda]}(x+(2 j-1-i))$. 
For example, let $\lambda=331$ and $\mu=21$. Then

$$
d_{\mu}(x)=(x-2) x(x+2)
$$

and $g_{\lambda}(x)=x(x-1)(x-2)(x+2)(x+1)$. So, $h_{\lambda, \mu}(x)=(x-1)(x+1)$.

The other extremal case is where $(\lambda, \mu)$ is $\mu$-extremal. In this case we only have a conjecture as to the roots of $\operatorname{det}\left(Z_{\lambda, \mu}(x)\right)$. Like the $\eta$-extremal case, the formula is remarkably simple.

Before stating this conjecture, it is helpful to compute the degree of $\operatorname{det}\left(Z_{\lambda, \mu}(x)\right)$. As noted in $\S 4$, the rows and columns of $Z_{\lambda, \mu}(x)$ are indexed by the 1 -factors on $2 k$ points. So the degree of $\operatorname{det}\left(Z_{\lambda, \mu}(x)\right)$ is $k$ times the number of 1 -factors on $2 k$ points. Since each 1 -factor has $k$ edges, one could hope to assign one root of $\operatorname{det}\left(Z_{\lambda, \mu}(x)\right)$ to every edge of each 1-factor on $2 k$ points.

Conjecture 6.7. Let $(\lambda, \mu)$ be an $\mu$-extremal pair. Let $\left\{\left(a_{i}, b_{i}\right): i=1,2, \ldots\right.$, $2 k\}$ be the coordinates of the squares of $[\lambda / \mu]$. For each edge $e=\{i, j\}$ in a 1 -factor on $2 k$ points, let $w(e)=\left(b_{i}-a_{i}\right)+\left(b_{j}-a_{j}\right)$. Then

$$
\operatorname{det}\left(Z_{\lambda, \mu}(x)\right)=\prod_{\delta \in B_{0, k}} \prod_{\begin{array}{c}
\text { edges } e \\
\text { of } \delta
\end{array}}(x+w(e)-1) .
$$

As an example of this, let $\lambda=32$ and $\mu=21$. Then the squares of $[\lambda / \mu]$ are $(1,3)$ and $(2,2)$. There is only one 1 -factor $\delta$ which joins these two points, and the unique edge $e$ of $\delta$ has weight $(3-1)+(2-2)=2$. So this conjecture predicts that

$$
\operatorname{det}\left(Z_{\lambda, \mu}(x)\right)=(x+1),
$$

which is indeed the case.

There is overwhelming computational evidence in support of this conjecture. Note in $\S 5$ the two columns marked $\mu$ and $\eta$. If the $\mu$ column is checked, that means the pair $(\lambda, \mu)$ is $\mu$-extremal. Likewise, if the $\eta$ column is checked, then $(\lambda, \mu)$ is $\eta$-extremal. Conjecture 6.7 holds for every pair on those lists which is $\mu$-extremal.

C. Sundaram roots. Certain of the roots of $\operatorname{det}\left(Z_{\lambda, \mu}(x)\right)$ are predicted by recent work of Sheila Sundaram [17]. Her work concerns the centralizer algebras $\operatorname{End}_{\mathrm{Sp}(2 n, \mathbb{R})}\left(T^{f} \mathbb{R}^{2 n}\right)$. One of her most elegant results gives an explicit combinatorial rule for describing the dimensions of the matrix rings in the Wedderburn decomposition of the algebra $\operatorname{End}_{\mathrm{Sp}(2 n, \mathbb{R})}\left(T^{f} \mathbb{R}^{2 n}\right)$. The next result follows by combining her results with earlier results of the authors.

Theorem 6.8. Let $\lambda$ and $\mu$ be partitions of $f$ and $m$, respectively, and let $\eta$ be an even partition. Suppose that $\phi$ is an L-R filling of $\left[\lambda^{\prime} / \mu^{\prime}\right]$ with content $\eta^{\prime}$, and suppose that some odd number $2 i+1$ occurs below row $n+i$ in $\phi$. If $2 n \geq l\left(\lambda^{\prime}\right)$ and $n \geq l\left(\mu^{\prime}\right)$, then $-(2 n)$ is a root of $\operatorname{det}\left(Z_{\lambda, \mu}(x)\right)$. 
Roots of $\operatorname{det}\left(Z_{\lambda, \mu}(x)\right)$ which are forced by Theorem 6.8 will be called Sundaram roots. In the data given in $\S 5$, the Sundaram roots are the starred roots.

As an example of Theorem 6.8 , consider $\lambda=62$ and $\mu=2$. There are two Littlewood-Richardson fillings of $\left[\lambda^{\prime} / \mu^{\prime}\right]$ with even content, namely:

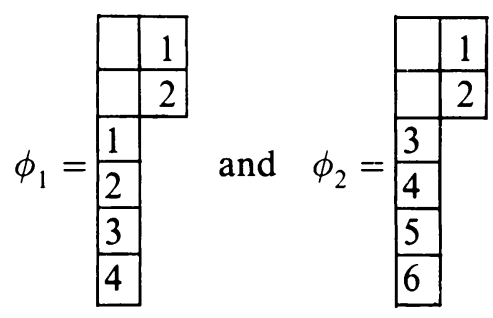

If $2 n \geq l(\lambda)$, then $n \geq 3$. It is easy to see that the hypotheses of Theorem 6.8 are never satisfied if $n \geq 4$. However, for $n=3$ the hypotheses are satisfied for $i=1$, since the 3 in filling $\phi_{1}$ occurs below row $4=(n+i)$. Hence, $(-6)$ is a Sundaram root for the pair $(62,2)$.

Theorem 6.8 is difficult to state and to apply, but it is potentially very important. Recall that the number of roots of $\operatorname{det}\left(Z_{\lambda, \mu}(x)\right)$ is $k$ times the number of Littlewood-Richardson fillings of $[\lambda / \mu]$ of even content. Theorem 6.8 gives a rule for deriving certain roots of $\operatorname{det}\left(Z_{\lambda, \mu}(x)\right)$ from the way the numbers are arranged in these fillings. One could hope for some more general combinatorial rule which would allow us to read $k$ roots from each Littlewood-Richardson filling of $[\lambda / \mu]$ of even content.

D. Hereditary roots. Perhaps the most striking pattern one sees in the tables of roots is the recursive structure given by the following theorem.

Theorem 6.9 (Hanlon and Wales). If $r$ is a root of $\operatorname{det}\left(Z_{\lambda, \mu}(x)\right)$ with multiplicity $\left(f_{\lambda} f_{\mu}\right) l$, and if $\hat{\lambda}$ is obtained from $\lambda$ by adding two squares not both in the same column, then the multiplicity of $r$ as a root of $\operatorname{det}\left(Z_{\hat{\lambda}, \mu}(x)\right)$ is at least $\left(f_{\hat{\lambda}} f_{\mu}\right) l$.

The proof of this theorem is difficult and involved. For the sake of brevity we will publish it elsewhere. However, we give an example of how Theorem 6.9 can be used.

Consider $(\hat{\lambda}, \mu)=(531,41)$. We see from Table 2 that the roots of $\operatorname{det}\left(Z_{\hat{\lambda}, \mu}(x)\right)$ are $3,0,-1,-3$, each with multiplicity $f_{\hat{\lambda}} f_{\mu}$. We can choose four different values of $\lambda$ to satisfy Theorem 6.9. In each case, $\operatorname{det}\left(Z_{\lambda, \mu}(x)\right)$ has exactly one root repeated $f_{\lambda} f_{\mu}$ times. These four choices of $\lambda$ and the corresponding roots are:

$\begin{array}{lc}421 & 3 \\ 43 & 0 \\ 52 & -3 \\ 51^{2} & -1\end{array}$


So Theorem 6.9 has predicted all four roots of $\operatorname{det}\left(Z_{\hat{\lambda}, \mu}(x)\right)$.

There is an obvious situation in which the roots of $\operatorname{det}\left(Z_{\hat{\lambda}, \mu}(x)\right)$ cannot be predicted by Theorem 6.9. This is the case where $\mu$ is a partition of $m$, and $\hat{\lambda}$ a partition of $m+2$. However, the following theorem handles that case.

Theorem 6.10. Suppose $[\hat{\lambda} / \mu]$ has size 2 with squares in positions $(a, b)$ and $(c, d)$. Then the unique root of $\operatorname{det}\left(Z_{\hat{\lambda}, \mu}(x)\right)$ is $1+(a-b)+(c-d)$.

Theorems 6.9 and 6.10 combine to yield a recursive method for obtaining some of the roots seen in the tables in $\S 5$. Roots obtained by this recursive method are called hereditary roots. There are very few nonhereditary roots for small values of $f$. Below we see a list of the nonhereditary roots for $f \leq 6$.

TABLE 3. Nonhereditary roots for $f \leq 6$.

$\begin{array}{rrrr}\lambda & \mu & \text { Nonhereditary roots } & \left(\lambda_{1}, \mu_{1}\right) \\ 4 & \phi & -2 & (3,1) \\ 2^{2} & \phi & 1 & (21,1) \\ 5 & 1 & -4 & (4,2) \\ 2^{2} 1 & 1 & 2 & \left(2^{2}, 2\right) \text { or }\left(21^{2}, 1^{2}\right) \\ 6 & \phi & -4 & (5,1) \\ 6 & 2 & -6 & (5,3) \\ 51 & 1^{2} & -4 & (41,21) \\ 42 & 2 & -1 & (41,3) \\ 3^{2} & 1^{2} & -1 & (32,21) \\ 2^{3} & \phi & 2 & \left(2^{2} 1,1\right) \\ 2^{3} & 2 & 3 & \left(2^{2} 1,21\right) \\ 2^{2} 1^{2} & 1^{2} & 3 & \left(2^{2} 1,21\right) \text { or }\left(21^{3}, 1^{3}\right)\end{array}$

The following conjecture is consistent with the data we have so far.

Conjecture 6.11. Let $\hat{\lambda}$ be a partition of $f$, and $\mu$ a partition of $m$. Suppose $r$ is a nonhereditary root of $\operatorname{det}\left(Z_{\hat{\lambda}_{, \mu}}(x)\right)$. Then there exists a pair $\left(\lambda_{1}, \mu_{1}\right)$ with $\lambda_{1}$ a partition of $f-1$ contained in $\lambda$ and $\mu_{1}$ a partition of $m+1$ containing $\mu$ and such that $r$ is a root of $\operatorname{det}\left(Z_{\lambda_{1}, \mu_{1}}(x)\right)$.

In the list of nonhereditary roots above we have indicated possible pairs $\left(\lambda_{1}, \mu_{1}\right)$ which satisfy Conjecture 6.11. At present, the authors have no idea why such a conjecture should be true, or how to find the pair $\left(\lambda_{1}, \mu_{1}\right)$.

\section{BIBLIOGRAPHY}

1. J. Birman and $\mathrm{H}$. Wenzl, Braids, link polynomials and a new algebra, preprint.

2. H. Boerner, Representations of groups with special consideration for the needs of modern physics, North-Holland, Amsterdam, 1970.

3. R. Brauer, On algebras which are connected with the semisimple continuous groups, Ann. of Math. (2) 38 (1937), 857-872.

4. C. Curtis and I. Reiner, Representation theory of finite groups and associative algebras, Pure and Applied Math., vol. 11, Wiley, New York, 1962. 
5. N. El Samra and R. King, Dimensions of irreducible representations of the classical Lie groups, J. Phys. A 12 (1979), 2317-2328.

6. W. Feit, Characters of finite groups, Benjamin, New York, 1967.

7. P. Hanlon and D. B. Wales, On the decomposition of Brauer's centralizer algebras, J. Algebra 121 (1989), 409-445.

8. $\ldots$, Eigenvalues connected with Brauer's centralizer algebras, J. Algebra 121 (1989), 446476.

9. G. James, The representation theory of the symmetric groups, Lecture Notes in Math., vol. 682, Springer-Verlag, Berlin and New York, 1978.

10. G. James and A. Kerber, The representation theory of the symmetric group, Encyclopedia of Mathematics and its Applications (G. C. Rota, ed.), Addison-Wesley, Reading, Mass., 1981.

11. V. F. R. Jones, Index for subfactors, Invent. Math. 72 (1983), 1-25.

12. W. Ledermann, Introduction to the theory of finite groups, 2nd ed., Oliver and Boyd, Edinburgh, 1953.

13. D. E. Littlewood, The theory of group characters, 2nd ed., Oxford Univ. Press, London, 1950.

14. I. G. Macdonald, Symmetric functions and Hall polynomials, Oxford Univ. Press, London, 1979.

15. J. Remmel and R. Whitney, Multiplying Schur functions, J. Algorithms 5 (1984), 471-487.

16. I. Schur, Ubber eine Klasse von Matrizen, die sich einer gegebenen Matrix zuordnen lassen, Dissertation, Berlin, 1901.

17. S. Sundaram, On the combinatorics of representations of $\operatorname{Sp}(2 n, \mathbb{C}), \mathrm{Ph} . \mathrm{D}$. thesis, M.I.T., 1986.

18. H. Wenzl, On the structure of Brauer's centralizer algebras, Ann. of Math. 128 (1988), 173-193.

19. H. Weyl, The classical groups, their invariants and representations, 2nd ed., Princeton Univ. Press, Princeton, N.J., 1946.

Department of Mathematics, University of Michigan, Ann Arbor, Michigan 481091003

Department of Mathematics, California Institute of Technology, Pasadena, CaliFORNIA 91125 